\title{
Localizing an Odor Source and Avoiding Obstacles: Experiments in a Wind Tunnel using Real Robots
}

\author{
Thomas Lochmatter, Nicolas Heiniger, and Alcherio Martinoli \\ Ecole Polytechnique Fédérale de Lausanne \\ Distributed Intelligent Systems and Algorithms Laboratory (DISAL) \\ \{thomas.lochmatter, alcherio.martinoli\}@epfl.ch
}

\begin{abstract}
We report on real-robot odor source localization experiments carried out in an environment with obstacles in the odor plume. The robot was equipped with an ethanol sensor and a wind direction sensor, and the experiments were carried out in a wind tunnel, i.e. in a controlled environment. An enhanced version of the surge-spiral algorithm was used, which was augmented with a dedicate behavior to manage obstacles (avoid them, or follow their contour). We compare the results in terms of distance overhead and success rate, and discuss the impact of obstacles on plume traversal.
\end{abstract}

\section{Introduction}

With the advances in robotics and chemicals sensor research in the last decade, odor sniffing robots have become an active research area. Notably the localization of odor sources would allow for very interesting robotic applications, such as search and rescue operations, safety and control operations on airports or industrial plants, and humanitarian demining [13, 2, 10, 4]. Many of these applications are time-critical, i.e. odor sources should be found as fast as possible. But as the structure of plumes in the air is intermittent in both time and space $[14,1]$, tracking plumes is a challenging problem.

In recent work, we carried out odor source localization experiments with bio-inspired algorithms both in simulation [7] and with real robots in a wind tunnel [8,9], and showed that upwind surge algorithms (namely, surge-spiral and surge-cast) are more effective than pure casting. In all experiments, the wind flow was laminar (i.e. low Reynolds number) and the plume therefore almost straight. In this paper, we discuss results of real-robot experiments carried out in the same setup, but with obstacles. Two types of obstacles were considered:

1. Surface obstacles, which have a negligible impact on the wind flow, but put constraints to where the robot can move.

2. Obstacles which both change the wind flow and constrain the robot movements. In this case, the robot not only has to deal with the obstacle, but also with the turbulence induced by it.

Only very few researchers have so far considered obstacles when studying odor source localization algorithms. Jatmiko et al. [5] carried out simulation experiments with three PSO-based algorithms endowed with collision avoidance to avoid running into obstacles. The authors compared the algorithm in environments with no, 5 and 10 surface obstacles. While their basic algorithm
(CPSO) suffered severely from putting obstacles, the algorithms using wind direction information (WU-I, WUII) did not show statistically significant performance losses when adding obstacles.

Other experiments with obstacles are described by Russell et al. in [11, Chapter 7] and [12]. Instead of avoiding obstacles, the authors propose to follow the obstacle contour (wall) until the plume is reacquired. Their wall following algorithm is based on 15 rules and to simplify dead reckoning, the robot only moves along four orthogonal directions (north, south, west, east). Russell et al. also discuss obstacles in conjunction with source declaration, since moving around an obstacle without reacquiring the plume on the other side means that the obstacle is the source.

The remainder of this paper is organized as follows: The experimental setup is described in Section 2, and the algorithms are presented in Section 3. Section 4 discusses the results.

\section{Experimental Setup}

The experimental setup was similar to the one used in our previous experiments without obstacles [8]. The experiments were thereby carried out in a $16 \mathrm{~m}$ long and $4 \mathrm{~m}$ wide wind tunnel with a wind flow of roughly $1 \mathrm{~m} / \mathrm{s}$ speed. This wind flow is laminar unless obstacles are placed. An odor source releasing a constant amount of ethanol vapor was placed in proximity of the wind inlet.

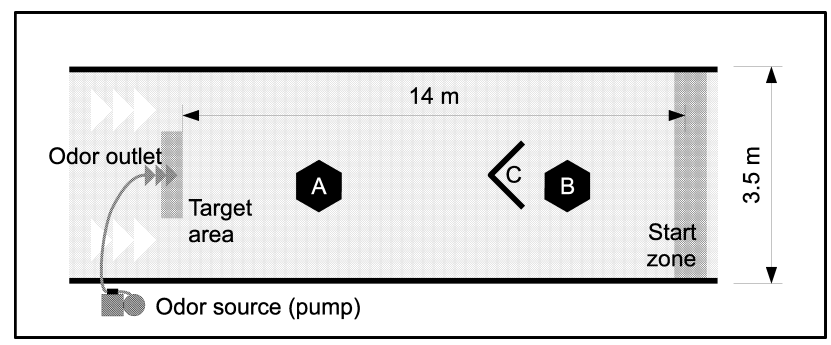

Figure 1: Sketch of the experimental setup with obstacles A, B, and C. Note that only one of the three obstacles was placed at a time.

We considered the three experimental configurations shown in Figure 1. Configuration (A) has a tall obstacle (changing wind flow) placed at $4 \mathrm{~m}$ downwind from the source. The shape of this obstacle can be described as a hexagon with an irregular border. Configuration (B) has the same obstacle, but placed $10 \mathrm{~m}$ downwind from the source. As the wind flow behind these obstacles was turbulent, the plume got very diluted in these areas. The robot both had difficulties measuring the wind direction and discriminating between plume and fresh air behind the obstacles. Configuration (C) consists of a V-shaped 
surface obstacle (leaving the wind flow unchanged) placed at $9 \mathrm{~m}$ downwind.

Pictures of these obstacles are shown in Figure 2, and the resulting plume in Figure 3.
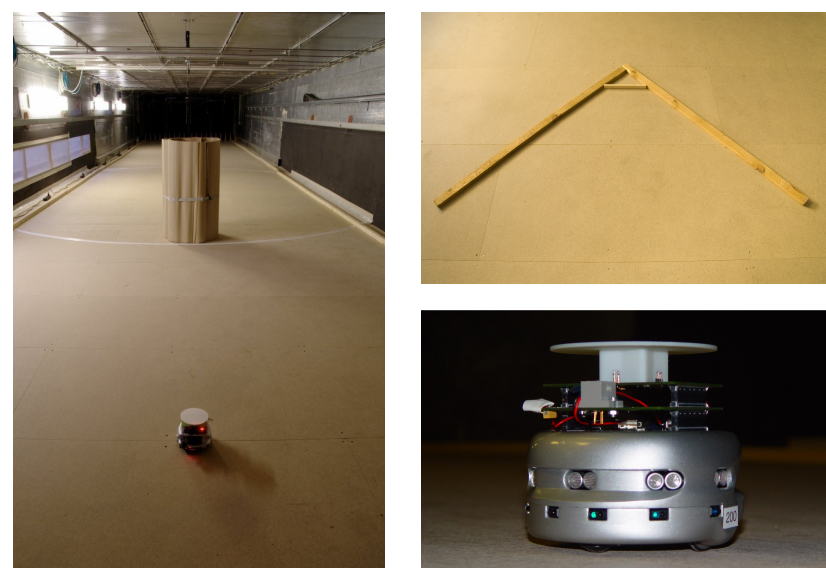

Figure 2: Left: wind tunnel with hexagonal obstacle at position B. Top right: V-shape obstacle (C). Bottom right: Khepera III robot.
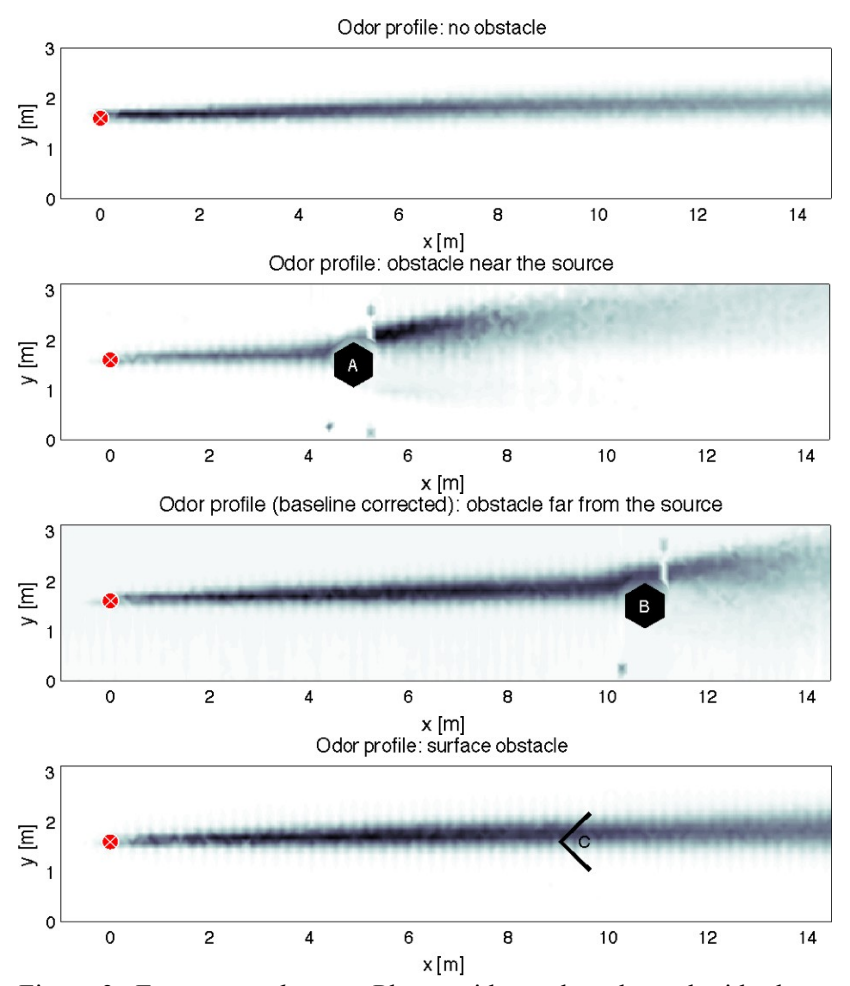

Figure 3: From top to bottom: Plume without obstacle, and with obstacles at positions A, B and C. Note that concentration levels are relative only the shape of the plumes can be compared. The plume maps were recorded by systematically scanning the wind tunnel (traversing system) with a MiCS-5521 sensor.

Our robotic platform is a Khepera III robot equipped with an odor sensor and a wind direction sensor board (see Figure 2). The odor sensor board mainly consists of a MiCS-5521 volatile organic compound sensor with a fast response time (approx. $0.1 \mathrm{~s}$ ). To take advantage of this response time, air was taken in and released with a small pump. The wind sensor board is based on 4 thermistors placed around a star-shape obstacle and can measure the wind direction with an accuracy of $10^{\circ}$ in laminar flow. The sensor also provides us with a confidence value, which drops significantly in turbulent flow.

\section{Algorithms}

The plume tracking strategy used here is based on the surge-spiral algorithm [8]. To deal with obstacles, we enhanced it with either a Braitenberg [6] obstacle avoidance or wall following algorithm using the 9 infrared sensors of the Khepera III robot. The left and right wheel speeds, $s_{l}$ and $s_{r}$, are thereby calculated as a linear combination of the raw infrared proximity sensor values, $p_{i}$, i.e.

$$
s_{l}=o_{l}+\sum_{i=1}^{9} w_{i} v_{i} \text { and } s_{r}=o_{r}-\sum_{i=1}^{9} w_{i} v_{i}
$$

and the weights, $w_{i}$, and bias speeds, $o_{l}$ and $o_{r}$, are chosen such that the resulting behavior is either obstacle avoidance or wall following.

With the obstacle avoidance algorithm, both the surge-spiral algorithm and the Braitenberg obstacle avoidance algorithm are running in parallel, and the output of the surge-spiral algorithm is simply the bias speed for the Braitenberg algorithm. As long as the robot is far away from any obstacle, the Braitenberg weights sum up to zero and leave the surge-spiral algorithm unmodified. When the robot is close to an obstacle, obstacle avoidance overrides surge-spiral.

With wall following, the surge-spiral algorithm is the only active algorithm in open space. When the robot approaches an obstacle, it switches to wall following and sticks to this mode until it has reached the other side of the obstacle. To find out when this has happened, the robot measures the wind direction in regular intervals and switches back to surge-spiral as soon as the wind is blowing towards the obstacle.

To deal with turbulence and dilute plume, the sensory input was processed as follows:

1. If the wind direction sensor returned a low confidence value for a measurement, that measurement was repeated and the confidence threshold decreased.

2. The variable odor threshold, $t_{i}$, was dynamically adjusted using the following additive-increasemultiplicative-decrease scheme:

$$
\begin{aligned}
& t_{i+1}=b_{i+1}+\delta \\
& b_{i+1}= \begin{cases}b_{i}+\alpha & \text { if } v_{i}>b_{i} \\
b_{i}(1-\beta)+v_{i} \beta & \text { otherwise }\end{cases}
\end{aligned}
$$

with $\alpha=0.01$ (experimentally found to be nearoptimal) and varying $\beta$ and $\delta$. While $\beta$ defines how fast the algorithm adapts to baseline changes, $\delta$ affects width of the plume, as perceived by the robot. $v_{i}$ denotes the raw measurement, while $b_{i}$ stands for the variable baseline.

Note that we only consider plume traversal and intentionally omit plume finding (i.e. randomized or systematic search until the plume is found) and source declaration (i.e. declaring that the source is in close vicinity), to 
prevent those two parts from interfering in the results. Hence, the robot starts in the plume, and source declaration is done by a supervisor (ideal source declaration). Experiments are considered successful when the robot has come in physical vicinity of the source, and unsuccessful if it bumped into an arena boundary.

We use the same two metrics as we did in our previous experiments [8]: the distance overhead, calculated as the traveled distance divided by the upwind distance, and the success rate, which is simply the fraction of successful runs.

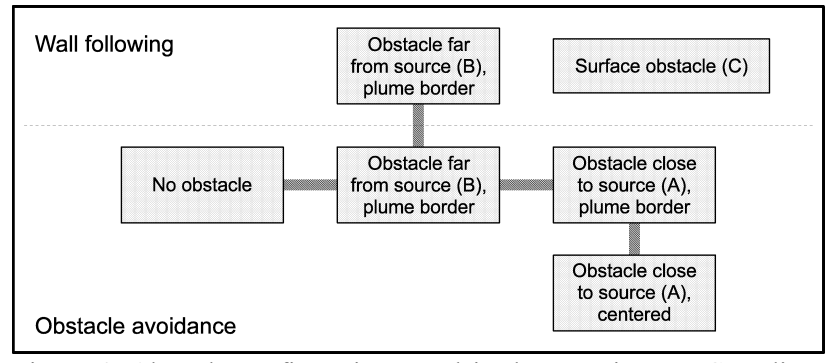

Figure 4: Obstacle configurations used in the experiments. Gray line connect configurations for which the results can be directly compared.

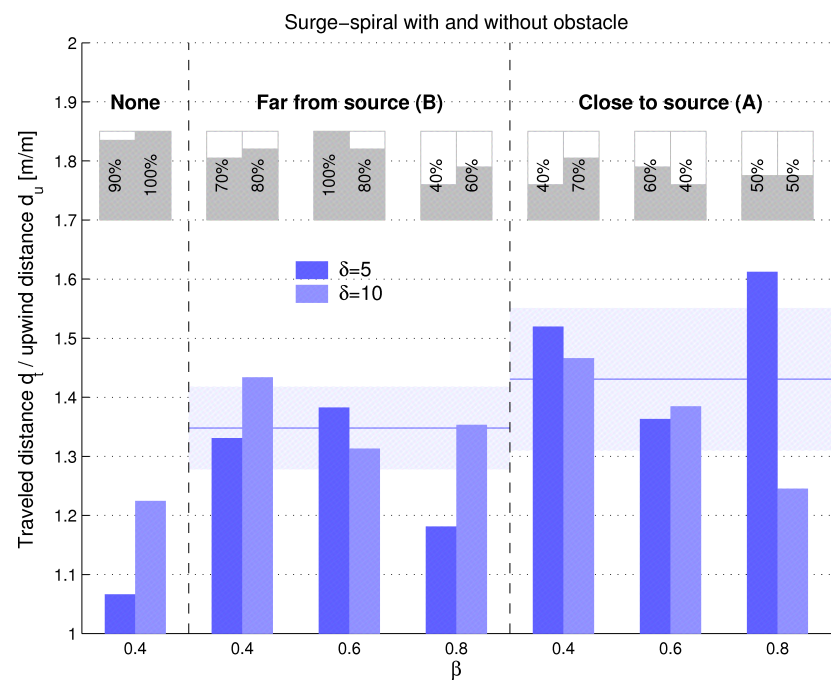

Figure 5: Comparison of runs with and without obstacles. The blue bars show the distance overhead (lower is better) and the grey bars indicate the success rate. The blue horizontal line is the mean for the group with the $95 \%$ confidence interval.

\section{Results and Discussion}

The different configurations we used to run our experiments and how they are related to each other are summarized in Figure 4.

\subsection{No Obstacle vs. Obstacles $A$ and $B$}

In the first series of runs, we compared the impact of obstacle $\mathrm{A}$ and $\mathrm{B}$ on the performance. 10 runs each were carried out for different values of $\beta$ and $\delta$, and the results plotted in Figure 5. The Braitenberg obstacle avoidance algorithm was used in all runs. In spite of the small number of runs carried out for each case, the results clearly show that the distance overhead increases and the success rate decreases when obstacles are present. Furthermore, there is evidence that the results are slightly worse in the case of obstacle A, which confirms our intuition that turbulent flow induces a performance penalty.

Even though the bars seem to suggest that higher $\beta$ values (i.e., faster threshold adaptation) yield lower distance overheads, there is statistically not enough evidence to support this statement.

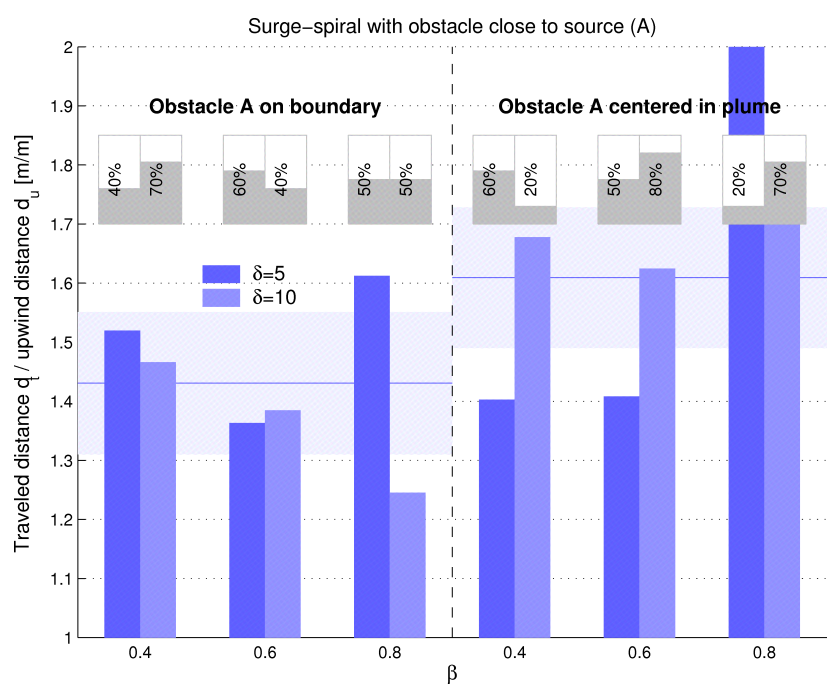

Figure 6: Effect of the position of the obstacle within the plume.

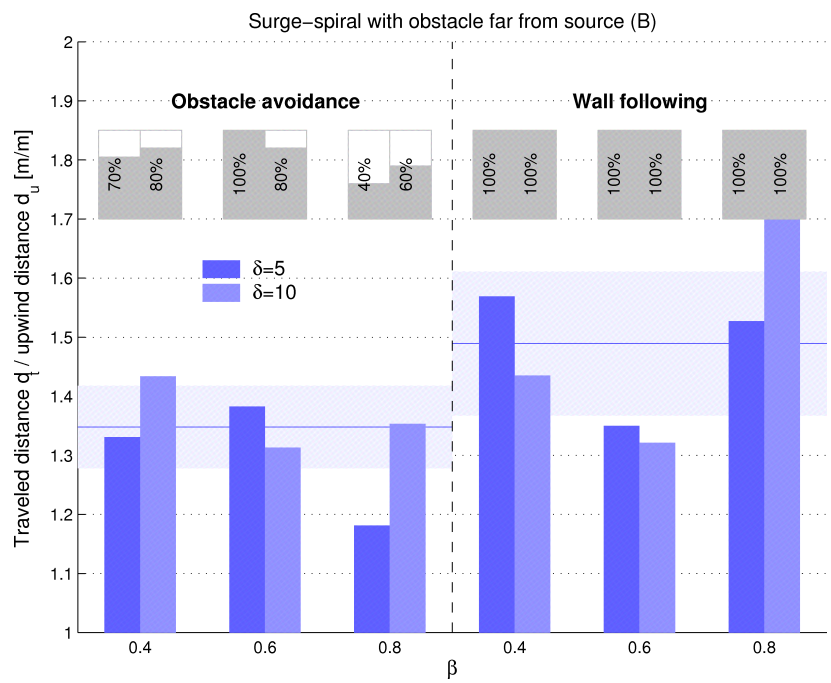

Figure 7: Comparison between obstacle avoidance and wall following.

\subsection{Obstacle A on Plume Boundary vs. in Center}

Figure 6 shows the impact of moving obstacle A from the plume boundary to the plume center, with the effect that the plume splits into two almost equal lobes behind the obstacle. It turns out that this has a negative effect on the distance overhead, while keeping the success rates at similar levels.

\subsection{Obstacle Avoidance vs. Wall Following}

Finally, we carried out experiments with the wall following algorithm. As shown in Figure 7, the success rate jumps to one, at the expense of a slightly higher distance overhead. 


\subsection{Surface Obstacles}

Braitenberg obstacle avoidance can perform poorly in case of non-convex obstacles. As sketched in Figure 8, the obstacle avoidance version of our algorithm is likely to get trapped in obstacle $\mathrm{C}$, while wall following is able to deal with it.

We carried out one set of 10 runs for $\beta=0.4$ and $\delta=10$ with both variants of the algorithm. While none of the obstacle avoidance runs succeeded, all wall following runs were successful with a mean distance overhead of 1.59.

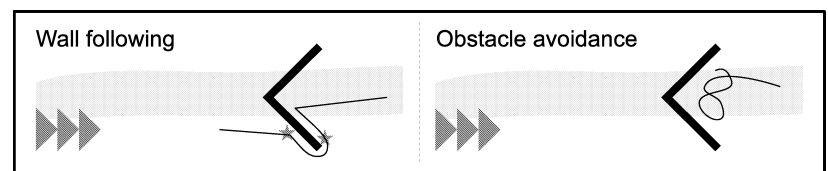

Figure 8: Wall following vs. Braitenberg obstacle avoidance with nonconvex obstacles.

\section{Conclusions}

We presented results of 280 odor source localization runs with a real robot in scenarios with various obstacles. Three main conclusions can be drawn from the experiments.

First, obstacles induce a penalty both because of the path constraints and the turbulence downwind the obstacle. The latter causes the plume to get diluted and become more peaky, but also affects the wind direction sensor accuracy which we have previously shown to have a big impact on the performance [7]. As turbulent flow is difficult to simulate with computer programs [1], real-robot experiments seem to be more suitable to study the effect of obstacles.

Second, both Braitenberg obstacle avoidance and wall following are able to deal with convex obstacles. Simple obstacle avoidance yielded a slightly better distance overhead in our experiments, but wall following scored with high success rates. Furthermore, for non-convex obstacles, wall-following is the preferred technique.

Finally, the surge-spiral algorithm seems to be a good candidate algorithm for plume traversal in complex environments. A few initial runs (not systematically recorded) with the casting algorithm [8] did not provide satisfactory results in our scenarios.

To our knowledge, this is the first systematic realrobot study on the performance of plume traversing algorithms in environments with obstacles. It is clear that the scenarios chosen here are not representative for the vast amount of potential real-world scenarios, and it may be too early to generalize the results presented here.

The effect of obstacles on odor source localization definitely needs to be studied further. Potential future research directions include studies with more than one obstacle, moving obstacles, or obstacles in immediate proximity of the source.

\section{References}

1. J. A. Farrell, J. Murlis, X. Long, W. Li, and R. T. Cardé. Filament-based atmospheric dispersion model to achieve short time-scale structure of odor plumes, Environmental Fluid Mechanics, vol. 2, 2002, p. 143169.

2. D. W. Gage. Many-Robot MCM Search Systems, Proceedings of the Autonomous Vehicles in Mine Countermeasures Symposium, April 1995, p. 9.56-9.64.

3. A. T. Hayes, A. Martinoli and R. M. Goodman. Distributed Odor Source Localization, IEEE Sensors Journal, vol. 2, no. 3, 2002, p. 260-271.

4. H. Ishida, T. Nakamoto, T. Moriizumi, T. Kikas, and J. Janata. Plume-Tracking Robots: A New Application of Chemical Sensors, Biological Bulletin, no. 200, April 2001, p. 222-226.

5. W. Jatmiko, K. Sekiyama, and T. Fukuda. A PSOBased Mobile Robot for Odor Source Localization in Dynamic Advection-Diffusion with Obstacles Environment, IEEE Computational Intelligence Magazine, May 2007, p. 37-51.

6. A. Lilienthal and T. Duckett. Experimental Analysis of Gas-Sensitive Braitenberg Vehicles, Advanced Robotics, vol. 18, no. 8, 2004, p. 817-834.

7. T. Lochmatter and A. Martinoli. Simulation Experiments with Bio-Inspired Algorithms for Odor Source Localization in Laminar Wind Flow, Proceedings of ICMLA 2008, p. 437-443.

8. T. Lochmatter and A. Martinoli. Tracking Odor Plumes in a Laminar Wind Field with Bio-Inspired Algorithms, Proceedings of ISER 2008, to appear.

9. T. Lochmatter, X. Raemy, L. Matthey, S. Indra, and A. Martinoli. A Comparison of Casting and Spiraling Algorithms for Odor Source Localization in Laminar Flow, Proceedings of ICRA 2008, p. 1138-1143.

10. M. Long, A. Gage, R. Murphy, and K. Valavanis. Application of the Distributed Field Robot Architecture to a Simulated Demining Task, Proceedings of ICRA 2005, p. 3193-3200.

11. R. A. Russell. Odour Detection by Mobile Robots, World Scientific Series in Robotics and Intelligent Systems, 1999.

12. R. A. Russell, D. Thiel, R. Deveza and A. MackaySim. A Robotic System to Locate Hazardous Chemical Leaks, Proceedings of ICRA 1995, p. 556-561.

13. G. S. Settles. Sniffers: Fluid-Dynamic Sampling for Olfactory Trace Detection in Nature and Homeland Security-The 2004 Freeman Scholar Lecture, Journal of Fluids Engineering, vol. 127, 2005, p. 189-218.

14. M. Vergassola, E. Villermaux, and B. I. Shraiman. 'Infotaxis' as a strategy for searching without gradients, Nature, vol. 445, January 2007, p. 406-409. 